\title{
Differential Mucosal IL-17 Expression in Two Gliadin-Induced Disorders: Gluten Sensitivity and the Autoimmune Enteropathy Celiac Disease
}

\author{
Anna Sapone ${ }^{a, b}$ Karen M. Lammers ${ }^{b}$ Giuseppe Mazzarellad Irina Mikhailenko ${ }^{c}$ \\ Maria Cartenì ${ }^{a}$ Vincenzo Casolaro $^{\text {b, e }}$ Alessio Fasano $^{b}$ \\ aSezione Biotecnologia e Biologia Molecolare, Dipartimento di Medicina Sperimentale, Seconda Università degli \\ Studi di Napoli, Naples, Italy; ${ }^{b}$ Mucosal Biology Research Center and ${ }^{c}$ Center for Vascular and Inflammatory \\ Diseases, University of Maryland School of Medicine, Baltimore, Md., USA; ${ }^{d}$ Istituto Scienze Alimentari, CNR, \\ Avellino, Italy; ${ }^{e}$ Division of Allergy and Clinical Immunology, Johns Hopkins University School of Medicine, \\ Baltimore, Md., USA
}

\section{Key Words}

Interleukin $17 \cdot$ Gliadin $\cdot$ Intestinal mucosa $\cdot$ Gluten

sensitivity $\cdot$ Celiac disease

\begin{abstract}
Background: The immune-mediated enteropathy, celiac disease (CD), and gluten sensitivity (GS) are two distinct clinical conditions that are both triggered by the ingestion of wheat gliadin. CD, but not GS, is associated with and possibly mediated by an autoimmune process. Recent studies show that gliadin may induce the activation of IL-17-producing $T$ cells and that IL-17 expression in the CD mucosa correlates with gluten intake. Methods: The small-intestinal mucosa of patients with CD and GS and dyspeptic controls was analyzed for expression of IL-17A mRNA by quantitative RT-PCR. The number of CD3+ and TCR- $\gamma \delta$ lymphocytes and the proportion of CD3+ cells coexpressing the Th17 marker CCR6 were examined by in situ small-intestinal immunohistochemistry. Results: Mucosal expression of IL-17A was significantly increased in CD but not in GS patients, compared to controls. This difference was due to enhanced IL-17A levels
\end{abstract}

in $>50 \%$ of $C D$ patients, with the remainder expressing levels similar to GS patients or controls, and was paralleled by a trend toward increased proportions of CD3+CCR6+ cells in intestinal mucosal specimens from these subjects. Conclusion: We conclude that GS, albeit gluten-induced, is different from $C D$ not only with respect to the genetic makeup and clinical and functional parameters, but also with respect to the nature of the immune response. Our findings also suggest that two subgroups of CD, IL-17-dependent and IL-17independent, may be identified based on differential mucosal expression of this cytokine. Copyright $\odot 2009$ S. Karger AG, Basel

\section{Introduction}

Gluten is the trigger of a heterogeneous set of conditions, including wheat allergy, gluten sensitivity (GS), and celiac disease (CD), that, combined, affect about $10 \%$ of the general population [1-3]. Once believed to fall exclusively into the domain of allergic conditions, e.g. wheat allergy, it is now clear that the intestinal and extraintes-

\section{KARGER}

๑ 2009 S. Karger AG, Basel

Fax +41613061234

E-Mail karger@karger.ch

www.karger.com
Accessible online at:

www.karger.com/iaa
Correspondence to: Prof. Alessio Fasano

University of Maryland School of Medicine

Mucosal Biology Research Center, Health Science Facility II

Rm. S345, 20 Penn St., Baltimore, MD 21201 (USA)

Tel. +1 410706 5501, Fax +1 410706 5508, E-Mail afasano@ mbrc.umaryland.edu 
tinal manifestations of $\mathrm{CD}$ are mediated by innate and adaptive immune pathways not shared with allergic disease $[1,4]$. CD, which roughly affects $1 \%$ of the general population, is considered an autoimmune disorder because of the presence of highly specific autoantibodies to tissue transglutaminase (tTG), and may lead to the onset of other autoimmune conditions [1]. Besides CD, GS may present with similar symptoms but without anti-tTG autoantibodies or autoimmune comorbidities [3]. CD is a life-long condition that requires rigorous and continuous avoidance of intake of gliadin, the toxic component of gluten, whereas GS can vary in terms of gluten threshold sensitivity and duration [5].

In support of the autoimmune, adaptive nature of $C D$, there is a strong genetic association with the MHC class II haplotype in CD patients, about $95 \%$ of whom carry HLA-DQ2, and the remainder HLA-DQ8 [6]. Conversely, only about $50 \%$ of patients with GS carry the HLADQ2 or -DQ8 haplotype, a percentage slightly higher than in the general population. The diversity of gluteninduced conditions suggests that the immune system reacts to and deals with the triggering environmental factor, e.g. gliadin, in distinct ways. The identification of interleukin (IL)-17-producing CD4+ T helper cells, Th17 cells, has had major impact on understanding immune processes not readily explained by the Th1/Th2 paradigm [7]. Aside from their possible involvement in host defense against microbes, Th17 cells have been linked to the pathogenesis of inflammatory and autoimmune diseases such as collagen-induced arthritis and colitis [7-9].

CD has been considered a classical Th1-mediated disorder because of the enhanced mucosal mRNA expression of interferon- $\gamma$, but not IL-4, in patients with untreated disease [10]. Following the identification of the Th17 $\mathrm{T}$ cell subset and the growing appreciation that these cells are centrally involved in the pathogenesis of autoimmune disorders, it has become important to investigate the possible involvement of Th17 cells in CD. A recent report by Castellanos-Rubio et al. [11] shows indeed that the expression of Th17-associated cytokines, e.g. IL-17A, is increased in patients with active CD as opposed to patients on a gluten-free diet. Furthermore, we have shown that gliadin can induce the production of the Th17-polarizing cytokines IL-1 $\beta$ and IL-23 in peripheral blood monocytes, providing a possible causative link between exposure to gluten and the expansion of IL-17-producing clones in $\mathrm{CD}$ [12].

As a preliminary effort to understand whether Th17 cells are uniquely involved in the pathogenesis of $\mathrm{CD}$ and hence, to further elucidate the autoimmune nature of this condition, we studied the expression of IL-17A in duodenal biopsies from CD and GS patients compared to controls.

\section{Materials and Methods}

\section{Patients}

Small-intestine biopsy samples were obtained from 13 active CD patients, 11 GS patients and 7 controls. All gave informed consent in accordance with a protocol approved by the Second University of Naples ethical committee. The subjects' characteristics are summarized in table 1. The diagnosis of CD or GS was based on the modified criteria from the European Society of Pediatric Gastroenterology, Hepatology and Nutrition (ESPGHAN) [13]. Patients were recruited based on the following inclusion criteria: gastrointestinal symptoms, such as chronic diarrhea, abdominal pain, constipation, weight fluctuation, bloating, weakness, fatty stools; extraintestinal symptoms, such as unexplained anemia, growth failure, joint pain, muscle cramps, osteoporosis, tingling numbness in the legs, tooth discoloration, glossitis, and autoimmune thyroiditis. Exclusion criteria for diagnosis of GS included seropositivity for anti-endomysium antibodies IgA and anti-tTG IgA, and high serum total and/or wheat-specific IgE. Other conditions characterized by altered intestinal permeability, e.g. type 1 diabetes, inflammatory bowel disease, Helicobacter $p y$ lori infection, and parasitosis, were also excluded. All subjects were tested for intestinal permeability in vivo using the lactulose/ mannitol double-sugar test as described [14].

\section{Methods}

Biopsy specimens were staged by histology according to the Marsh classification [15]. Intraepithelial lymphocytes (IELs) were counted by immunohistochemistry as described [16]. Mucosal CD3+ lymphocytes coexpressing the Th17 marker CCR6 were visualized by confocal microscopy with a Radiance 2100 system (Carl Zeiss MicroImaging, Thornwood, N.Y., USA) upon double labeling with rabbit anti-CD3 (Sigma-Aldrich, St. Louis, Mo., USA), mouse anti-CCR6 (clone 11A9; BD Biosciences, San Diego, Calif., USA), and secondary fluorochrome-conjugated antibodies (Invitrogen Molecular Probes ${ }^{\circledR}$, Eugene, Oreg., USA) as described [17]. Total RNA was extracted from biopsy specimens using Trizol (Invitrogen, Grand Island, N.Y., USA), and reverse transcribed to cDNA using the First Strand cDNA Synthase Kit (MBI Fermentas, Hanover, Md., USA) with random hexamer primers. Realtime quantitative PCR was performed with the Applied Biosystems 7500 Fast Real-Time PCR System using the Taqman protocol (Applied Biosystems, Foster City, Calif., USA). Primers and probes for the evaluation of IL-17A (Hs00936345_m1), and for RNA loading and integrity control, 18S (Hs99999901_s1) expression were purchased from Applied Biosystems. The data were calculated as the $\Delta_{\mathrm{CT}}$ of IL17A to $18 \mathrm{~S}$ reactions, and are expressed as percent of $18 \mathrm{~S}\left(2^{-\Delta C T} \times 100\right)$. Differences between groups were tested for significance using the Mann-Whitney and KruskalWallis nonparametric algorithms. 
Table 1. Clinical and laboratory characteristics of the study subjects

\begin{tabular}{|c|c|c|c|}
\hline & Controls & $\mathrm{CD}$ & GS \\
\hline Samples, $\mathrm{n}$ & 7 & 13 & 11 \\
\hline Age at diagnosis, years & $29.1 \pm 5.1$ & $35.2 \pm 3.3$ & $28.9 \pm 4.4$ \\
\hline $\operatorname{Sex}(F / M)$ & $2 / 5$ & $11 / 2$ & $7 / 4$ \\
\hline Intestinal symptoms & dyspepsia & $\begin{array}{l}\text { chronic diarrhea } \\
\text { abdominal pain } \\
\text { weight fluctuation } \\
\text { weakness } \\
\text { smelly, fatty stool }\end{array}$ & $\begin{array}{l}\text { diarrhea } \\
\text { abdominal pain } \\
\text { weight fluctuation } \\
\text { gas }\end{array}$ \\
\hline $\begin{array}{l}\text { Extraintestinal } \\
\text { symptoms }\end{array}$ & none & $\begin{array}{l}\text { bone or joint pain } \\
\text { osteoporosis } \\
\text { behavioral changes } \\
\text { tingling leg numbness } \\
\text { muscle cramps } \\
\text { missed menstrual periods } \\
\text { infertility } \\
\text { recurrent miscarriage } \\
\text { delayed growth } \\
\text { tooth discoloration } \\
\text { unexplained anemia }\end{array}$ & $\begin{array}{l}\text { bone or joint pain } \\
\text { osteoporosis } \\
\text { leg numbness } \\
\text { muscle cramps } \\
\text { unexplained anemia } \\
\text { glossitis } \\
\text { thyroiditis }\end{array}$ \\
\hline EMA-IgA & all negative & 12 positive $(92 \%)$ & all negative \\
\hline tTG-IgA & all negative & 11 positive $(85 \%)$ & all negative \\
\hline AGA-IgA/IgG & all negative & 7 positive $(54 \%)$ & 5 positive $(46 \%)$ \\
\hline MHC profile & 2 DQ2 and/or DQ8 (29\%) & 12 DQ2 and/or DQ8 (92\%) & 4 DQ2 and/or DQ8 (36\%) \\
\hline Wheat IgE & not tested & all negative & all negative \\
\hline LA/MA test & normal $(<0.030)$ & altered $(>0.030)$ & normal $(<0.030)$ \\
\hline
\end{tabular}

EMA = Anti-endomysium antibodies; AGA = anti-gliadin antibodies; $\mathrm{MHC}=$ major histocompatibility complex; LA/MA = lactulose/mannitol.

\section{Results and Discussion}

All subjects underwent upper-duodenal endoscopy. Those with GS revealed normal to mildly inflamed mucosa (Marsh 0-1), while all CD patients showed partial or subtotal villous atrophy with crypt hyperplasia according to the ESPGHAN criteria [13]. Figure 1 shows representative photomicrographs of CD3 staining on mucosal biopsy specimens from dyspeptic control patients, activeCD patients, and GS patients. As expected, CD patients (fig. 1c) had increased numbers of CD3+ IELs ( $>50 / 100$ enterocytes) compared to controls (fig. 1a), while GS patients had a number of CD3+ IELs intermediate between $\mathrm{CD}$ patients and controls in the context of rela- tively conserved villus architecture (fig. 1b). The numbers of TCR- $\gamma \delta$ IELs were only elevated in CD subjects ( $>3.4 / 100$ enterocytes), while in GS patients the numbers of $\gamma \delta$ IELs were similar to those in controls. More details on these findings are provided elsewhere [Sapone et al., manuscript in preparation].

As shown in figure 2, IL-17A gene expression, measured by real-time quantitative PCR in biopsy specimens, was significantly elevated in active CD patients (mean \pm SE: $0.0088 \pm 0.002 \%$ of 18 S) compared to GS patients $(0.0025 \pm 0.0008 \% ; \mathrm{p}=0.03)$ and controls $(0.0022 \pm 0.0013 \% ; \mathrm{p}=0.024)$. The level of variance across the groups was also statistically significant $(\mathrm{p}<$ 0.025 ). Interestingly, as figure 2 shows, this difference 

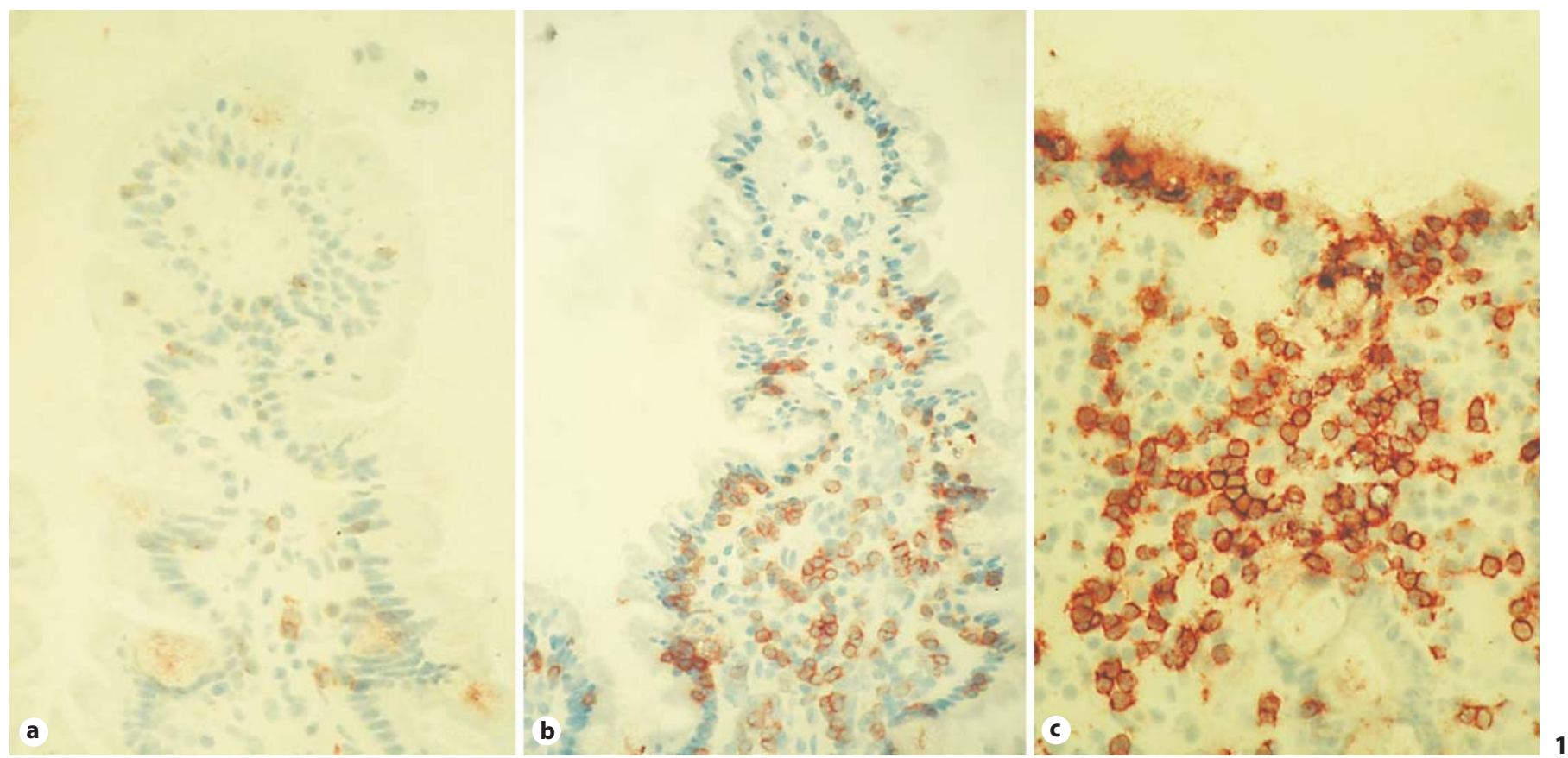

Fig. 1. Representative microphotographs of mucosal CD3 expression in a dyspeptic control patient (a), a gluten-sensitive (GS) patient (b) and a celiac disease (CD) patient with active disease (c) (40× magnification).

Fig. 2. Mucosal IL-17A mRNA expression in the 3 groups. IL-17A expression is significantly increased in CD patients $(n=13)$ compared to controls $(\mathrm{n}=7 ; \mathrm{p}=0.024)$ and GS patients $(\mathrm{n}=11 ; \mathrm{p}=$ $0.03)$.

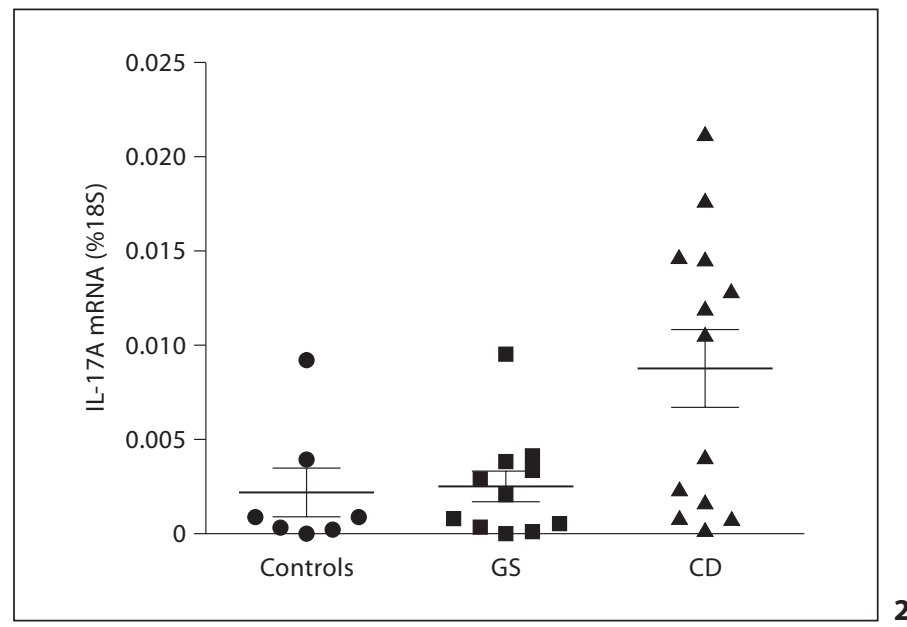

appeared to be sustained by a subgroup (7 out of 13) of $\mathrm{CD}$ patients. The significance of this finding is at present unclear, as this subgroup did not seem to differ from the other CD patients with respect to clinical, histological, and serological parameters or the MHC haplotypes, with the exception of a trend toward increased positivity for anti-gliadin antibodies. Immunofluorescence staining of intestinal mucosal sections from this IL$17^{\text {high }}$ subgroup documented an increased percentage $(35.6 \pm 3.7 \%)$ of $\mathrm{T}$ cells expressing the constitutive Th17 surface marker CCR6 relative to IL-17 ${ }^{\text {low }}$ CD $(24.8 \pm$ $8.1 \%)$ or control groups $(24.0 \pm 3.6 \%$; a typical staining

is shown in fig. 3), but these differences did not reach statistical significance, presumably due to the small sample size and a high degree of interindividual variability. It can be speculated that this variability might reflect different stages of the condition, or imply an as yet unappreciated level of heterogeneity in the immune mechanisms involved in CD pathogenesis. Longitudinal studies in larger populations might help elucidate these issues.

Together, these data show that the expression of IL-17, a cytokine that is thought to be involved in inflammatory and autoimmune processes $[8,9,12]$, is elevated in 

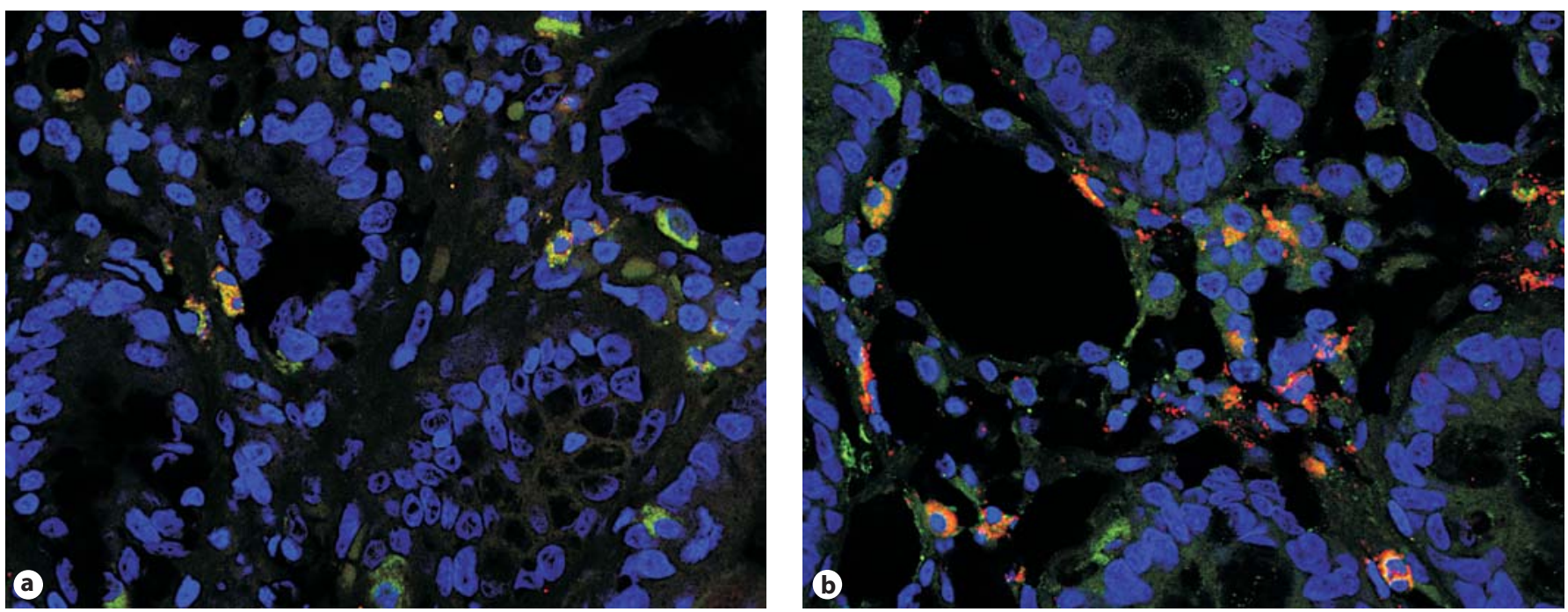

Fig. 3. Representative immunofluorescence staining of mucosal CD3 (FITC; green) and CCR6 (Alexa Fluor ${ }^{\circledR}$ 568 ; red) in a control subject (a) and a CD patient expressing high levels of mucosal IL-17A (b). Shown are merged confocal microscopy images $(60 \times$ magnification) in which cells expressing both markers appear yellow. Nuclei were counterstained in blue with TO-PRO ${ }^{\circledR}-3$ (Molecular Probes).

CD but not in GS. Our work confirms and extends the study by Castellanos-Rubio et al. [11] showing gluten-dependent expression of IL-17A in active CD. In addition, combined with the other observed clinical and histological differences, it further supports the idea that $\mathrm{CD}$ and GS are distinct entities and that the immune system deals with gluten in different ways, possibly depending on the genetic makeup $[2,3,18]$. Where in GS as well as in wheat allergy the gluten-induced response leads to immunity toward a non-self diet component, i.e. gliadin, in CD a deviated, self-directed adaptive response leads, in addition, to the onset of a full-fledged autoimmune process. In clear contrast to GS, CD results from a complex, as yet undetermined, interplay of increased intestinal permeability, mucosal damage, environmental factors additional to gluten, and genetic predisposition, which involves both MHC and non-MHC genes [Sapone et al., manuscript in preparation] [6]. Based on our recent work, we can speculate that gliadin may directly affect the expansion of Th17 clones by inducing the expression of IL-23 among other cytokines [12]. Studies are needed to elucidate whether the unique involvement of these cells in $\mathrm{CD}$ versus GS reflects differential expansion in response to gliadin-induced factors versus other mechanisms, including mucosal recruitment.

In conclusion, here we present for the first time evidence of differential intestinal mucosal immune respons- es to gluten between CD and GS, further supporting the notion that $\mathrm{CD}$ is the only clinical form of gluten reactivity involving autoimmune mechanisms.

\section{Acknowledgements}

The authors wish to thank Dr. R. Maarten Egeler for helpful advice, Ms. Molly Hritzo and Dr. Davide Libreri for technical help and scientific discussions. This study was supported in part by National Institutes of Health grant DK-48373 (to A.F.). A.S., K.M.L., G.M., I.M., M.C., and V.C. have no relationships to declare. A.F. is stock holder of ALBA Therapeutics (Baltimore, Md., USA).

References

Int Arch Allergy Immunol 2010;152:75-80
Catassi C, Fasano A: Celiac disease. Curr Opin Gastroenterol 2008;24:687-691.

-2 Anderson LA, McMillan SA, Watson RG, Monaghan P, Gavin AT, Fox C, Murray LJ: Malignancy and mortality in a populationbased cohort of patients with coeliac disease or 'gluten sensitivity'. World J Gastroenterol 2007;13:146-151.

-3 Ferguson A, Gillett H, Humphreys K, Kingstone K: Heterogeneity of celiac disease: clinical, pathological, immunological, and genetic. Ann N Y Acad Sci 1998;859:112-120. 
4 Constantin C, Huber WD, Granditsch G, Weghofer M, Valenta R: Different profiles of wheat antigens are recognised by patients suffering from coeliac disease and IgE-mediated food allergy. Int Arch Allergy Immunol 2005; 138:257-266.

$\checkmark 5$ Catassi C, Fabiani E, Iacono G, D’Agate C, Francavilla R, Biagi F, Volta U, Accomando S, Picarelli A, De Vitis I, Pianelli G, Gesuita R, Carle F, Mandolesi A, Bearzi I, Fasano A: A prospective, double-blind, placebo-controlled trial to establish a safe gluten threshold for patients with celiac disease. Am J Clin Nutr 2007;85:160-166.

6 Monsuur AJ, Wijmenga C: Understanding the molecular basis of celiac disease: what genetic studies reveal. Ann Med 2006;38:578591.

77 Weaver CT, Hatton RD, Mangan PR, Harrington LE: Il-17 family cytokines and the expanding diversity of effector T cell lineages. Annu Rev Immunol 2007;25:821-852.

$>8$ Murphy CA, Langrish CL, Chen Y, Blumenschein W, McClanahan T, Kastelein RA, Sedgwick JD, Cua DJ: Divergent pro- and antiinflammatory roles for IL-23 and IL-12 in joint autoimmune inflammation. J Exp Med 2003;198:1951-1957.
Yen D, Cheung J, Scheerens H, Poulet F, McClanahan T, McKenzie B, Kleinschek MA, Owyang A, Mattson J, Blumenschein W Murphy E, Sathe M, Cua DJ, Kastelein RA, Rennick D: IL-23 is essential for T cell-mediated colitis and promotes inflammation via IL-17 and IL-6. J Clin Invest 2006;116:13101316.

10 Nilsen EM, Jahnsen FL, Lundin KE, Johansen FE, Fausa O, Sollid LM, Jahnsen J, Scott $\mathrm{H}$, Brandtzaeg P: Gluten induces an intestinal cytokine response strongly dominated by interferon gamma in patients with celiac disease. Gastroenterology 1998;115:551563.

11 Castellanos-Rubio A, Santin I, Irastorza I, Castano L, Carlos Vitoria J, Ramon Bilbao J: Th17 (and Th1) signatures of intestinal biopsies of CD patients in response to gliadin. Autoimmunity 2009;42:69-73.

12 Harris KM, Fasano A, Mann DL: Cutting edge: IL-1 controls the IL-23 response induced by gliadin, the etiologic agent in celiac disease. J Immunol 2008;181:4457-4460.

13 Troncone R, Bhatnagar S, Butzner D, Cameron D, Hill I, Hoffenberg E, Maki M, Mendez V, de Jimenez MZ: Celiac disease and other immunologically mediated disorders of the gastrointestinal tract: Working Group report of the second World Congress of $\mathrm{Pe}$ diatric Gastroenterology, Hepatology, and Nutrition. J Pediatr Gastroenterol Nutr 2004 39(suppl 2):S601-S610.
14 Sapone A, de Magistris L, Pietzak M, Clemente MG, Tripathi A, Cucca F, Lampis R, Kryszak D, Carteni M, Generoso M, Iafusco D, Prisco F, Laghi F, Riegler G, Carratu R, Counts D, Fasano A: Zonulin upregulation is associated with increased gut permeability in subjects with type 1 diabetes and their relatives. Diabetes 2006;55:1443-1449.

15 Green PH, Rostami K, Marsh MN: Diagnosis of coeliac disease. Best Pract Res Clin Gastroenterol 2005;19:389-400.

16 Mazzarella G, Stefanile R, Camarca A, Giliberti P, Cosentini E, Marano C, Iaquinto G, Giardullo N, Auricchio S, Sette A, Troncone R, Gianfrani C: Gliadin activates HLA class I-restricted CD8+ T cells in celiac disease intestinal mucosa and induces the enterocyte apoptosis. Gastroenterology 2008;134:10171027.

17 Annels NE, Da Costa CE, Prins FA, Willemze A, Hogendoorn PC, Egeler RM: Aberrant chemokine receptor expression and chemokine production by Langerhans cells underlies the pathogenesis of Langerhans cell histiocytosis. J Exp Med 2003;197:13851390.

18 Catassi C, Fasano A: Is this really celiac disease? Pitfalls in diagnosis. Curr Gastroenterol Rep 2008;10:466-472. 